\title{
Renal Toxicity
}

National Cancer Institute

\section{Source}

National Cancer Institute. Renal Toxicity. NCI Thesaurus. Code C115459.

Toxicity that impairs or damages the kidney. This condition is often caused by the administration of a pharmaceutical agent that causes damage to the kidney. 